\title{
Elective mechanical cardiopulmonary support during transcatheter aortic valve implantation
}

Iritakenishi T.1 ${ }^{1}$ Imada T. ${ }^{1}$, Okitsu K. ${ }^{1}$, Iwasaki M. ${ }^{1}$, Maeda K. ${ }^{2}$, Fujino Y. ${ }^{1}$

${ }^{1}$ Osaka University Graduate School of Medicine, Dept of Anaesthesiology \& Intensive Care, Suita, Japan,

${ }^{2}$ Osaka University Graduate School of Medicine, Dept of Cardiovascular Surgery, Suita, Japan

\section{Background}

Although transcatheter aortic valve implantation (TAVI) is widely known as a low invasive treatment for aortic stenosis (AS), intraoperative hemodynamic changes due to surgical steps may be fatal to patients with severely impaired cardiac function.

For extremely high-risk cases, we have used mechanical cardiopulmonary support (CPS) electively ${ }^{[1]}$. In order to avoid circulatory collapse due to rapid pacing or aortic insufficiency after pre-dilatation, CPS was usually started before pre-dilatation and gradually discontinued after valve deployment.

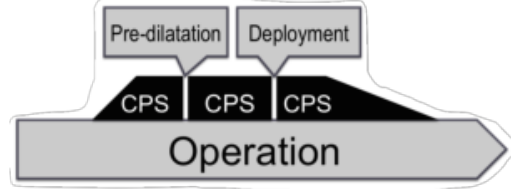

In this report, we aimed to assess the efficacy of elective CPS during TAVI.

\section{Methods}

Consecutive patients who underwent TAVI for severe AS between October 2009 and August 2015 in our institute were enrolled into the study, and were divided into two groups; patients who required elective CPS and those who did not.

The preoperative (patient characteristics and echocardiographic findings), operative data were compared between the two groups.

Statistic analysis was conducted by the Students' t-test or the Wilcoxon rank-sum test or the chi-square test. A $P$ value of $<0.05$ was considered statistically significant.

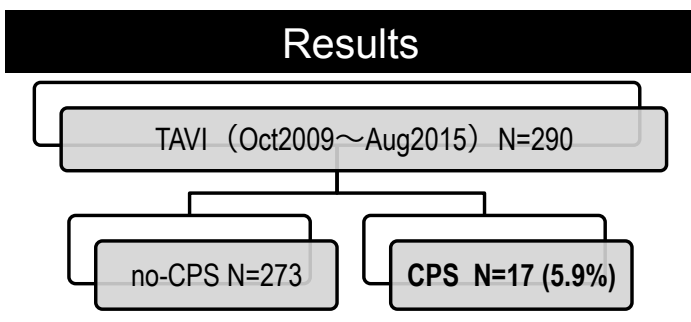

Reasons for elective CPS N

Impaired left ventricular function

14 cases

Presereved LVEF

but probable intraoperative myocardial ischemia due to diffuse coronary stenosis

Presereved LVEF

but severe heart failure

with pulmonary hypertension

2 cases

\begin{tabular}{|c|c|c|c|}
\hline Patients Characteristics & no-CPS (N=273) & CPS (N=17) & $\mathbf{P}$ \\
\hline Age $(y r)^{*}$ & $82.9(5.89)$ & $80.2(8.69)$ & N.S. \\
\hline Female sex & $63.6 \%$ & $64.7 \%$ & N.S. \\
\hline $\operatorname{BSA}\left(m^{2}\right)^{*}$ & $1.45(0.17)$ & $1.38(0.16)$ & N.S. \\
\hline Diabetes & $30.1 \%$ & $18.8 \%$ & N.S. \\
\hline Coronary artery disease & $40.9 \%$ & $56.3 \%$ & N.S. \\
\hline History of cardiovasc.surgery & $17.7 \%$ & $43.8 \%$ & $<0.05$ \\
\hline Peripheral vascular disease, & $37.9 \%$ & $31.3 \%$ & N.S. \\
\hline $\mathrm{BNP}(\mathrm{pg} / \mathrm{dL})$ * & $442.8(703.3)$ & $1395.6(1459.6)$ & $<0.05$ \\
\hline NYHA> III , (\%) & $51.3 \%$ & $82.4 \%$ & $<0.05$ \\
\hline Logistic euroSCORE (\%) * & $22.1(13.0)$ & $59.1(29.7)$ & $<0.05$ \\
\hline Preoperartive Echocardiogram & no-CPS (N=273) & CPS (N=17) & $\mathbf{P}$ \\
\hline $\operatorname{LVDd}^{*}(\mathrm{~mm})$ & $46.3(7.60)$ & $52.0(8.41)$ & $<0.05$ \\
\hline $\operatorname{LVEF}^{*}(\%)$ & $63.2(12.0)$ & $39.7(18.2)$ & $<0.05$ \\
\hline peak $\Delta \mathrm{P}^{*}(\mathrm{mmHg})$ & $86.1(29.9)$ & $71.2(23.0)$ & $<0.05$ \\
\hline $\mathrm{AVA}^{*}\left(\mathrm{~cm}^{2}\right)$ & $0.69(0.18)$ & $0.53(0.17)$ & $<0.05$ \\
\hline TR $>$ moderate & $6.9 \%$ & $23.5 \%$ & $<0.05$ \\
\hline Surgical data & no-CPS (N=273) & CPS (N=17) & $\mathbf{P}$ \\
\hline Transapical approach & $45.1 \%$ & $29.4 \%$ & N.S. \\
\hline Postope. peak CK-MB $(\mathrm{ng} / \mathrm{mL})^{\dagger}$ & $13.3(7.2-19.7)$ & $12.3(5-14.7)$ & N.S. \\
\hline Postope. hospital stay (day) ${ }^{\dagger}$ & $10(8-14)$ & $15(10-21.5)$ & $<0.05$ \\
\hline Home discharge, $\mathrm{n}(\%)$ & $249(91.2 \%)$ & $12(70.6 \%)$ & $<0.05$ \\
\hline 30-days mortality, n (\%) & $3(1.1 \%)$ & $1(5.9 \%)$ & N.S. \\
\hline
\end{tabular}

\section{Discussions}

$\checkmark$ The patients with elective CPS had significantly higher risk profiles than those without CPS.

$\checkmark$ Although postoperative hospital stay was longer and home discharge rate was lower in the CPS group, 30-days mortality did not show significant difference between two groups, and the postoperative outcomes of the CPS group were considered to be satisfactory.

\section{Limitations}

Lack of information about anesthetic technique and use of hemodynamic drugs, which would have given good insights to anesthetic management.

\section{Conclusion}

Elective CPS during TAVI is considered to be effective to perform a safe operation for AS patients at extremely high risk.

\section{References}

1. Maeda K, et al. On-pump transcatheter aortic valve replacement in patients with poor left ventricular function. J Card Surg. 2012 Nov;27(6):686-8 